\title{
INTRAORAL VS EXTEAORAL APPROACH IN THE MANAGEMENT OF MANDIBULAR ANGLE FRACTURE, A RANDOMIZED CONTROLLED CLINICAL TRIAL
}

\author{
Saleh Ahmed Bakry* and Heba M. Kamel ${ }^{* *}$
}

\begin{abstract}
Objective: To compare between the intraoral and extraoral approaches in the management of mandibular angle fractures.

Methodology: This randomized controlled clinical trial was conducted in the Faculty of Dentistry, Cairo University. The study comprised patients with mandibular angle fractures who were divided in two equal groups: Intraoral Group (A) and Extraoral group (B). Patients were followed up post-operatively after 7 days, 14 days, 2 months and 6 months respectively. Patients were followed up to examine the interincisal distance by using a caliper. motor function of the facial nerve using House-Brackmann grading system. Also, the surgical time needed for incision and dissection was recoded intraoperatively.
\end{abstract}

Results: A total of 14 patients with mandibular angle fracture were presented into the study. The age ranged from 18 to 50 years with mean age for group (A) $(34.28 \pm 11.65)$ while for group (B), it was $(39.85 \pm 20.61)$ with no significant difference between both groups $(\mathrm{P}=0.545)$. No significant difference was found between the two groups as regarding the mouth opening. Facial nerve function was reserved more in the intraoral group with no significant difference in all follow up intervals. Regarding time needed for incision and reflection measure in minutes, group (A) had a significantly lower time than group $(\mathrm{B})$ which was $(\mathrm{P}<0.001)$. However, for the time needed for ORIF there was no significant difference between both group $(\mathrm{P}=0.198)$.

Conclusion: In the management of mandibular angle fractures, no difference was found in the mouth opening between extraoral and intraoral approach. As regarding the facial nerve injury, it was found that the facial nerve damage was higher in the extraoral approach but with no difference between both groups. However, our study showed statistically lower time needed for incision and reflection in the intraoral than the extraoral approach.

KEYWORDS: Mandibular angle fracture, Intraoral approach, extraoral approach, Facial nerve.

* Associate Professor, Oral and Maxillofacial Surgery Department, Faculty of Dentistry, Cairo University 


\section{INTRODUCTION}

The facial trauma is considered to be very common worldwide, two thirds of which are mandibular fractures. The mandibular angle fractures represent about $30 \%$ of all mandibular fractures ${ }^{(1-3)}$. The most common causes of this type of fractures are motor vehicle accidents and assaults. ${ }^{(4)}$

The frequent involvement of the angular fractures could be attributed to many anatomical causes, such as: 1) the cross-sectional area of bone in this part of the mandible is diminished due to the more medial convergence of the alveolar bone, 2) the common presence of the unerupted or impacted wisdom tooth leads to weakening in the area of the angle, 3) the sudden change in bone path between the ramus and body in the angle area leads to increased stresses. ${ }^{(5)}$

Duo to the unique position of the angle area and the difficulty in obtaining good access via the surgical approaches, the management of mandibular angle fractures could be considered to be very challenging and technique sensitive when compared to other fractures of the mandible. ${ }^{(6)}$ Also, it has the highest recorded rates of postoperative complications of any area of the mandible. ${ }^{(4)}$

Among the literature, still there is wide controversy regarding the best treatment of mandibular angle fractures. However, the current treatment protocols for this type of fractures include open reduction and rigid internal fixation in addition to intraoperative maxillomandibular fixation (MMF) due to stability obtained with this type of treatment, this allows for the primary bone healing in association with limited immediate postoperative function. ${ }^{(7)}$

Several approaches for the exposure and reduction of the fractures in the angular area have been mentioned in the literature. This included transoral and extra oral approaches. ${ }^{(8)}$ The proper decision in selecting the appropriate surgical approach depends on many factors, such as: amount of fracture displacement, number of the fractured segments and whether it is associated with other facial fractures or not, accessibility to the fractured segments, perpendicular drilling for plate fixation, and the complications related to each approach. ${ }^{(9)}$ The aim of this study is to compare between the intraoral and extraoral approaches in the management of mandibular angle fractures. Different factors such as the mouth opening, facial nerve integrity, and the time needed for incision and reflection for both approaches have been assessed.

\section{PATIENTS AND METHODES}

\section{Study Design}

This study was a randomized controlled clinical trial conducted on 14 patients divided in two groups (seven patients in each group). The study was performed in the Faculty of Dentistry, Cairo University. The Ethics Committee of the Faculty of Dentistry, Cairo University approved the protocol, and a detailed informed written consent including the details of surgery and the possible complications was obtained from all patients.

\section{The patients included in the study fulfilled the following criteria}

\section{Inclusion Criteria}

- Patients with unfavorable displaced mandibular angle fractures and intact facial nerve at the fracture side.

- Patients free from any systemic diseases that interfere with normal bone healing.

- No sex or gender predilection.

- $\quad$ Age range (18-50).

\section{Exclusion criteria}

- Patients with any degree of affection of the facial nerve motor function.

- Patients with favorable non-displaced mandibular angle fractures.

- Patients with Comminuted fragmented \& infected angle fractures. 


\section{Randomization}

This study was a randomized clinical controlled trial. Patients were randomly assigned into two equal groups: group (A) and group (B) according to the website (http//www.random.org.eg). Each group consisted of 7 patients.

\section{Patient grouping:}

The selected patients fulfilled the inclusion criteria and randomly allocated to two groups.

\section{Group A (Intraoral approach group):}

This group consists of seven cases with mandibular angle fractures which treated with ORIF using intraoral approach.

\section{Group B (Extraoral approach group):}

This group consists of seven cases with mandibular angle fractures which treated with ORIF using the conventional Risdon (periangular) approach.

\section{Preoperative Preparation}

At the initial visit, all patients underwent a clinical and dental history to avoid any of the exclusion criteria mentioned above. History of trauma including date and time of injury; direction of force or any circumstances as bleeding or loss of consciousness were collected from each patient. Trauma survey clearance was performed by the trauma team to inspect any soft tissues laceration; edema; ecchymosis; mandible contour deformity, facial asymmetry and any other facial bones injury. The integrity of the neurosensory and motor function of the injured area; the amount of occlusal discrepancy with complete examination of the dentition and the mandibular movements were also checked to determine the maximal Interincisal opening and midline shift in opening and closing positions. Digital panoramic radiographs were obtained for evaluation (Figure 1) as a primary survey. All patients underwent multislice CT scan to determine the amount of displacement and compare it with the postoperative CT scan to assess the integrity of reduction and fixation (Figure 2 and 3 ).

\section{Surgical techniques}

The necessary laboratory investigations were requested from all the patients including $\mathrm{CBC}$, coagulation profile, random blood sugar level, liver function test, kidney function test and chest x-ray. Then the preanesthetic evaluation were performed. All the patients were treated as inpatients under general anesthesia.

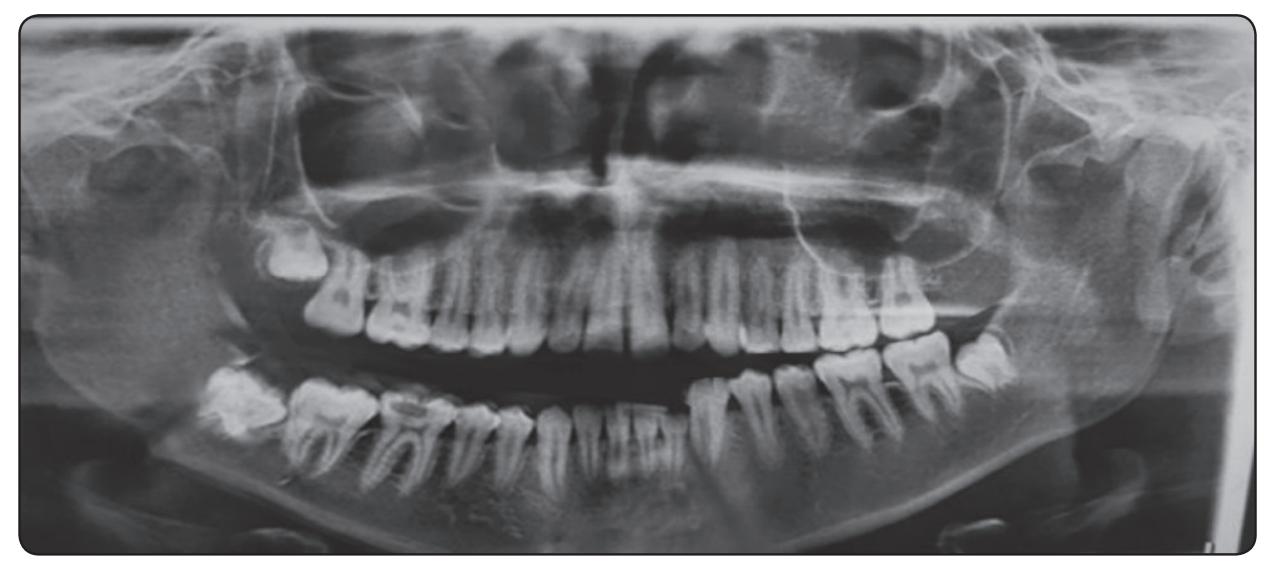

Fig. (1): Panoramic x-ray showing right mandibular angle fracture. 


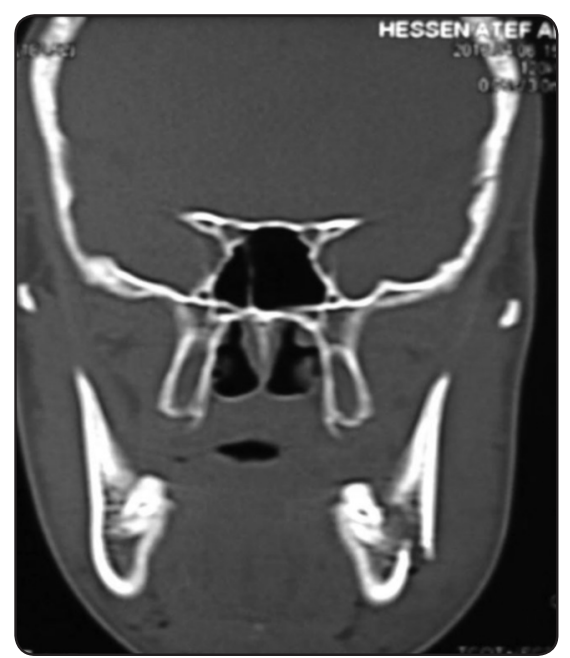

Fig. (2): Preoperative coronal cut showing left fractured mandibular angle.

For group (A), an intraoral incision was carried out after the injection of local anesthetic solution with 1:200000 epinephrine (Epinephrine: Misr Pharmaceutical Industries, A.R.E) to expose the anterior border of ramus, buccal cortex \& external oblique ridge to obtain good surgical access to the fracture line. Intraoperative IMF was performed to obtain proper occlusion then the two miniplates were adapted to follow the contour of the buccal cortex (superior and inferior border plating). After fracture fixation, the IMF was released, and the occlusion was checked for stability \& reproducibility. Wound closure was performed using vicryl 3/0 intraorally (Figure 4).

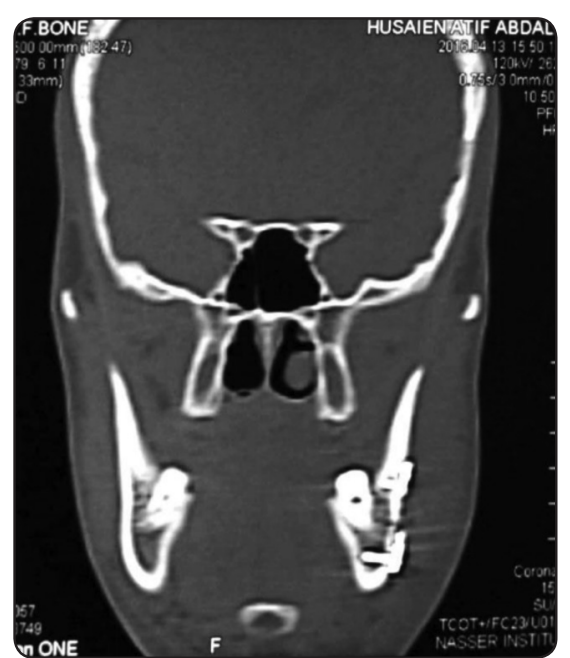

Fig. (3): Postoperative coronal cut showing the angle fracture after reduction and fixation.

For group (B), periangular incision was used to access the surgical field. Incision and dissection continued through SMAS, parotidomasseteric fascia and platysma muscle to expose the mandibular angle area. Intraoperative IMF was performed to obtain proper occlusion then the mandibular angle fracture was reduced and fixed using internal rigid fixation. After fracture fixation, the IMF was released, and the occlusion was checked for stability \& reproducibility. Closure of the wound was performed in a layered fashion first, the pterygomandibular sling and the platysmal layer using $3 / 0$ vicryl then the skin closed using 5/0 prolene suture (Figure 5).

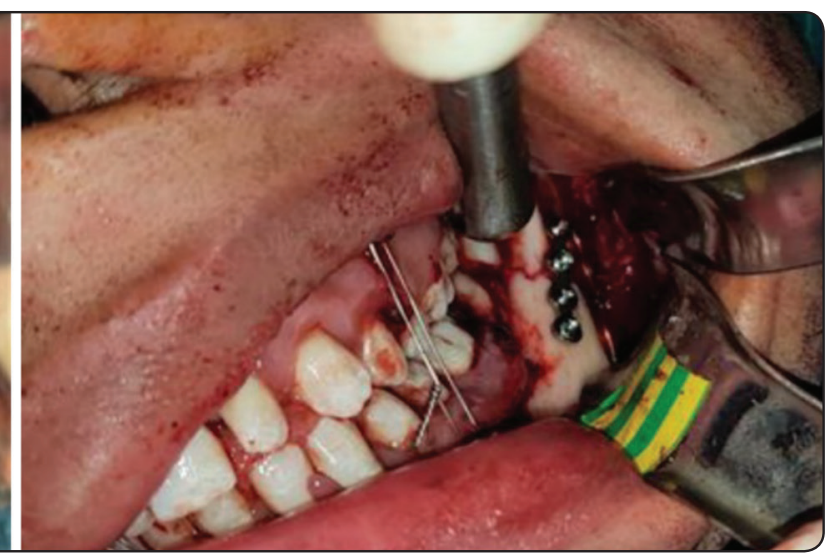

Fig. (4): A photo showing transoral approach, fracture line and plate fixation. 

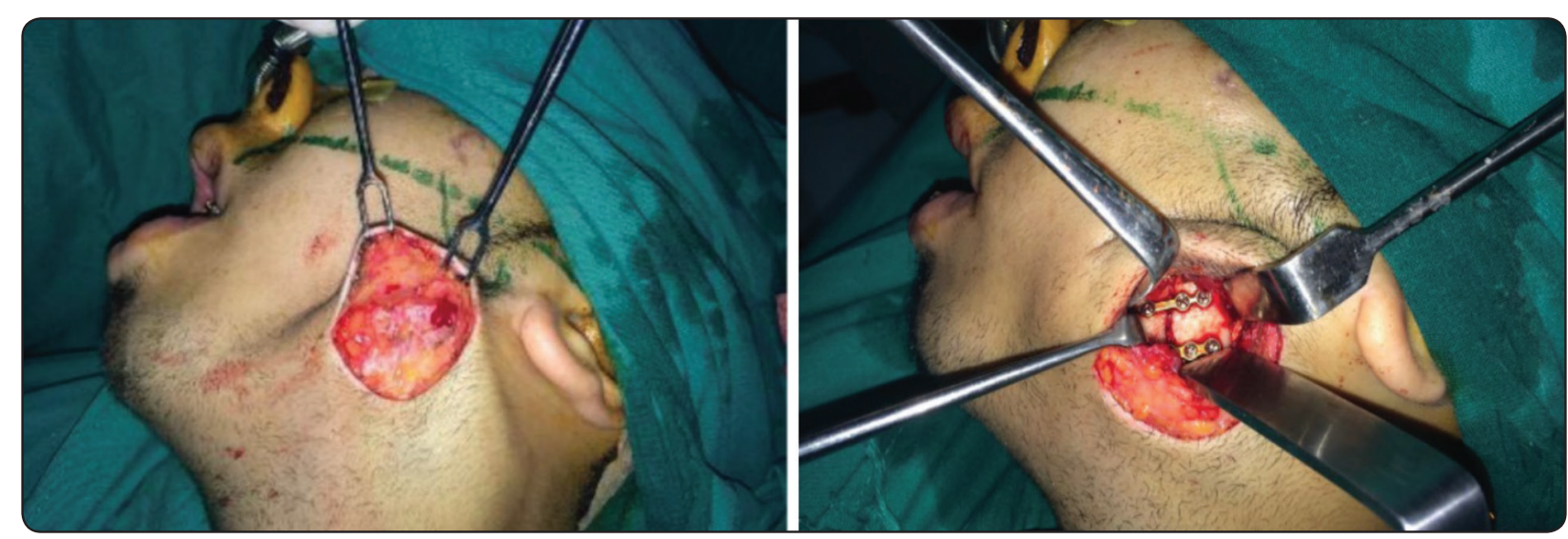

Fig. (5): A photo showing extraoral approach, fracture line and plate fixation.

\section{Postoperative care:}

Postoperatively all patients had intravenous antibiotics (Unasyn $1500 \mathrm{mg}$ vials) each twelve hours for five days and analgesics (Voltaren $75 \mathrm{mg}$ ampoule) three times daily for three days. A strict oral hygiene measures were given to all the patients using chlorohexidine mouth wash five times daily wash five times daily for 1 week.

\section{Clinical follow up parameters:}

Patients were followed up post-operatively after 7 days, 14 days, 2 months and 6 months respectively. Patients were followed up to examine the motor function of the facial nerve using HouseBrackmann ${ }^{(10)}$ grading system and the interincisal distance by using a caliper. Also, the surgical time needed for making incision and dissection was recorded intraoperatively.

\section{Method of assessment:}

\section{Assessment of the mandibular movements:}

The measurement of mouth opening was based on House-Brackmann (10) classification which is presented by the following table (Table 1).

\section{Assessment of facial nerve function:}

Examination of the facial nerve integrity was performed by asking the patient to perform certain movements of the facial muscles as whistling and ballooning of the check to examine the facial nerve branches. This method of evaluation was based on House-Brackmann Facial Nerve Grading System ${ }^{(10)}$ which is presented by the following table (Table 2).

TABLE (1): House-Brackmann ${ }^{(10)}$ classification of mouth opening

\begin{tabular}{ll}
\hline Grade 0 & Maximum mouth opening $>40 \mathrm{~mm}$ \\
\hline Grade 1 & Maximum mouth opening $\leq 40 \mathrm{~mm}$ \\
\hline Grade 2 & Maximum mouth opening $\leq 35 \mathrm{~mm}$ \\
\hline Grade 3 & Maximum mouth opening $<30 \mathrm{~mm}$ \\
\hline
\end{tabular}

\section{Statistical analysis}

Collected data were presented as frequencies (n) and percentages (\%) and were analyzed using chi square test. Parametric data were analyzed using independent t-test. Nonparametric data were analyzed using Mann Whitney U test for intergroup comparisons. The significance level was set at $\mathrm{P} \leq$ 0.05 for all tests. Statistical analysis was performed with IBM ${ }^{\circledR}$ SPSS $^{\circledast}$ (SPSS Inc., IBM Corporation, NY, USA) Statistics Version 25 for Windows. 
TABLE (2): House-Brackmann Facial Nerve Grading System for facial nerve integrity:

\begin{tabular}{cccl}
\hline Grade & & At rest & \\
\hline I & Normal & Symmetry & Normal facial function \\
\hline II & Mild dysfunction & Normal symmetry and & Forehead: moderate to good function \\
& & tone & $\begin{array}{l}\text { Eye: complete closure with minimum effort } \\
\text { Mouth: slight asymmetry }\end{array}$ \\
\hline III & Moderate dysfunction & Normal symmetry and & Forehead: slight to moderate movement \\
& & tone & Eye: complete closure with effort \\
& & & Mouth: slight weakness with maximum effort \\
\hline IV & Moderately severe & Normal symmetry and & Forehead: none \\
& dysfunction & tone & Eye: incomplete closure \\
& & & Mouth: asymmetric with maximum effort \\
\hline V & Severe dysfunction & Asymmetry & Forehead: none \\
& & & Eye: incomplete closure \\
& & Asymmetry & No movement \\
\hline VI & Absence of function & Aslight movement \\
\hline
\end{tabular}

\section{RESULTS}

A total of 14 patients with mandibular angle fracture were presented into the study. The age ranged from 18 to 50 years with mean age for group (A) $(34.28 \pm 11.65)$ while for group (B), it was (39.85 \pm 20.61$)$ and there was no significant difference between both groups $(\mathrm{P}=0.545)$.

\section{Clinical evaluation:}

\section{1-Mouth opening:}

Mouthopening was measuredinmm.Immediately post-operative, mouth opening was only limited to $(23.57 \pm 2.63)$ in group $(\mathrm{A})$ and $(25.42 \pm 4.64)$ in group (B) with no significant difference between both groups $(\mathrm{P}=0.376)$. After 4 weeks there was a significant increase in both groups to $(32.00 \pm 2.64)$ and $(36.71 \pm 2.92)$ respectively with group (B) being significantly higher $(\mathrm{P}=0.008)$. After 6 months there was a significant increase of mouth opening mean value in group (A) to $(39.00 \pm 1.91)$ while in group (B), it increased to $(39.85 \pm 1.06)$ with no significant difference between both groups $(\mathrm{P}=0.321)$ (Table 3).
TABLE (3): Mean and standard deviation (SD) values for the mouth opening $(\mathrm{mm})$ in both groups.

\begin{tabular}{cccc}
\hline \multirow{2}{*}{$\begin{array}{c}\text { Follow-up } \\
\text { intervals }\end{array}$} & \multicolumn{2}{c}{ Mouth opening (Mean \pm SD) } & \multirow{2}{*}{ P-value } \\
\cline { 2 - 3 } & Group A & Group B & \\
\hline Immediate & $23.57 \pm 2.63$ & $25.42 \pm 4.64$ & 0.376 \\
\hline 4 weeks & $32.00 \pm 2.64$ & $36.71 \pm 2.92$ & $0.008^{*}$ \\
\hline 6 months & $39.00 \pm 1.91$ & $39.85 \pm 1.06$ & 0.321 \\
\hline P-value & $<0.001^{*}$ & $<0.001^{*}$ & \\
\hline
\end{tabular}

*; significant ( $p \leq 0.05) n s ;$ non-significant ( $p>0.05)$

\section{Facial nerve affection}

At baseline and follow-up intervals, there was no significant difference in facial nerve affection in both groups and during the follow-up intervals $(\mathrm{P}>0.05)$. According to House-Brackmann grading scale ${ }^{(10)}$ the majority of patients of both groups had a score of (1) (85.7\% and $57.1 \%)$ respectively. Only one patient had a score of (2) in the group (B), while number of patients with score (3) was (1) in group (A) and (2) in group (B). After 3 months, one patient from each group changed from score (3) to score (2). After 6 months all patients of group (A) had score 
TABLE (4): Frequencies (n) and percentages (\%) for House-Brackmann scores in both groups.

\begin{tabular}{|c|c|c|c|c|c|c|}
\hline \multirow{2}{*}{$\begin{array}{l}\text { Follow-up } \\
\text { intervals }\end{array}$} & \multirow[t]{2}{*}{ Housebrackmann score } & \multicolumn{2}{|c|}{ Group (A) } & \multicolumn{2}{|c|}{ Group (B) } & \multirow[t]{2}{*}{ P-value } \\
\hline & & $\%$ & (n) & $\%$ & (n) & \\
\hline \multirow[t]{3}{*}{ Immediate } & Score (1) & $85.7 \%$ & (6) & $57.1 \%$ & (4) & \multirow{3}{*}{0.420} \\
\hline & Score (2) & $0 \%$ & $(0)$ & $14.3 \%$ & (1) & \\
\hline & Score (3) & $14.3 \%$ & (1) & $28.6 \%$ & (2) & \\
\hline \multirow[t]{3}{*}{3 months } & Score (1) & $85.7 \%$ & (6) & $71.4 \%$ & (5) & \multirow{3}{*}{0.580} \\
\hline & Score (2) & $14.3 \%$ & (1) & $14.3 \%$ & (1) & \\
\hline & Score (3) & $0 \%$ & $(0)$ & $14.3 \%$ & (1) & \\
\hline \multirow[t]{3}{*}{6 months } & Score (1) & $100 \%$ & (7) & $85.7 \%$ & (6) & \multirow{3}{*}{0.299} \\
\hline & Score (2) & $0 \%$ & (0) & $14.3 \%$ & (1) & \\
\hline & Score (3) & $0 \%$ & (0) & $0 \%$ & (0) & \\
\hline \multirow[t]{4}{*}{12 months } & Score (1) & $100 \%$ & (7) & $85.7 \%$ & (6) & \multirow{3}{*}{0.299} \\
\hline & Score (2) & $0 \%$ & (0) & $14.3 \%$ & (1) & \\
\hline & Score (3) & $0 \%$ & $(0)$ & $0 \%$ & $(0)$ & \\
\hline & P-value & & & & & \\
\hline
\end{tabular}

*; significant $(p \leq 0.05) n s ;$ non-significant $(p>0.05)$

(1) while one patient of group (B) had score (2). After 12 months, the status remained unchanged. (Table 4)

\section{3-Operative time}

Mean and standard deviation (SD) values for operative time (minutes) in group (A) was $(5.71 \pm 1.79)$ and it had a significantly lower skin to bone time (incision and dissection) time than group (B) which was $(23.14 \pm 4.52)(\mathrm{P}<0.001)$. As regarding the time for ORIF there was no significant difference between both group $(\mathrm{P}=0.198)$. (Table 5)

TABLE (5): Mean and standard deviation (SD) values for operative time (minutes) in both groups

\begin{tabular}{cccc}
\hline \multirow{2}{*}{ Operative time } & \multicolumn{2}{c}{ Operative time $($ Mean \pm SD) } & \multirow{2}{*}{ P-value } \\
\cline { 2 - 3 } & Group (A) & Group (B) & \\
\hline Skin to bone & $5.71 \pm 1.79$ & $23.14 \pm 4.52$ & $<0.001^{*}$ \\
\hline ORIF & $20.28 \pm 4.15$ & $23.14 \pm 3.67$ & 0.198 \\
\hline
\end{tabular}

*; significant $(p \leq 0.05) n s ;$ non-significant $(p>0.05)$

\section{DISCUSSION}

Mandibular angle fractures are one of the most common fractures encountered in the maxillofacial region. Mandibular angle fractures have been described a lot in the literature, however, only a limited number of studies have mentioned the surgical approaches used to treat such a challenging fracture $^{(1-3)}$.

Treatment of these types of fractures ranges from simple maxillomandibular fixation to rigid internal fixation of the bony segments. The right choice of the surgical approach is considered mandatory for the successful fixation method. (2) The traditional and standard approach used for mandibular angle fractures was the extraoral approach. However, due to the current increase in patients' esthetic demands and the need to avoid the extraoral scar, the intraoral approach has gained popularity since it was first reported by Kazanjian in 1933. ${ }^{(11)}$ 
It was obvious that among the published literature, no single approach has been proved to be the best choice for treating mandibular angle fractures and still there are wide controversies regarding favorable and unfavorable fractures at the mandibular angle region. ${ }^{(12)}$

Age range in our study ranged from 18 to 50 years with a mean age of 34.28 years in group (A) and 39.85 years in group (B). This range was near to the age range found is another retrospective study with mean age of patients about 31.57 years. ${ }^{(13)} \mathrm{A}$ possible explanation is that this age group which is the young adults, are more exposed to facial and mandibular trauma because this large mass of population participates more in activities either for travelling or working. ${ }^{(14)}$

The mean interincisal opening was measured to the speed of recovery and the time needed to return to the normal function in each group. Our study revealed that both groups had limitation in mouth opening immediately postoperative. However, both groups regained a reasonable mouth opening during their follow up intervals with no significant difference between the two groups. The limitation in the mouth opening at the early follow up intervals could be attributed to the soft tissue dissection, surgical edema, and pain and after the resolution of these factors, the mouth opening has returned to its normal range. ${ }^{(8)}$

As regarding the facial nerve affection, our study showed that the preservation of the facial nerve was better in the intraoral approach than the extraoral approach, with no difference between the two groups throughout the follow up intervals. This was in contrary to other studies who found that extraoral approach was associated with higher damage to the facial nerve. ${ }^{(15,16)}$ However, our results were in acceptance with other studies who found that the facial nerve damage was higher in the extraoral approach but with no statistical difference between the two approaches. ${ }^{(17)}$
As regarding the operative time, our study showed statistically lower time needed for incision and reflection in the intraoral than the extraoral approach. This finding was in accordance with other studies. ${ }^{(9)}$ This result seems logic and could be explained by the need for more careful dissection, preservation of the facial nerve and ligation of facial vessels when compared to the transoral approach. (1,8, $9,18,19)^{-1}$

\section{CONCLUSION}

In the management of mandibular angle fractures, no difference was found in the mouth opening between extraoral and intraoral approach. As regarding the facial nerve injury, it was found that the facial nerve damage was higher in the extraoral approach but with no difference between the two approaches. However, our study showed statistically lower time needed for incision and reflection in the intraoral than the extraoral approach.

\section{REFERENCES}

1. Bilal Y, Rahim AU, Gul SM, Warraich RA. Outcomes of extra oral versus intraoral approach for Mandibular angle fracture reduction. J Pak Med Assoc. 2020 Dec;70 (12 (A):2088-2091. doi: 10.47391/JPMA.673. PMID: 33475577

2. Ebenezer V, Balakrishrian KM, Sivakumar. Comparison of intraoral and extra oral approach for the management of angle fracture under general anesthesia. World J Med Sci. 2014; 10: 317-8.

3. Rashid A, Eyeson J, Haider D, van Gijn D, Fan K. Incidence and patterns of mandibular fractures during a 5-year period in a London teaching hospital. Br J Oral Maxillofac Surg. 2013 Dec;51(8):794-8. doi: 10.1016/j.bjoms.2013.04.007. Epub 2013 Jun 2. PMID: 23735734.

4. Perez R, Oeltjen JC, Thaller SR. A review of mandibular angle fractures. Craniomaxillofac Trauma Reconstr. 2011 Jun; 4(2): 69-72. doi: 10.1055/s-0031-1272903. PMID: 22655117 ; PMCID: PMC3193298.

5. Darwich A, darwich khaldoun, al-shurbaji mhd. 3D Finite Element Analysis of Miniplate Fixation Techniques in Mandibular Angle Fractures. J Indian Dent Assoc. 2016; 9 (12): 10 . 
6. Cillo JE Jr, Ellis E 3rd. Management of bilateral mandibular angle fractures with combined rigid and nonrigid fixation. J Oral Maxillofac Surg. 2014 Jan;72(1):106-11. doi: 10.1016/j.joms.2013.07.008. Epub 2013 Sep 14. PMID: 24045187.

7. Gear AJ, Apasova E, Schmitz JP, Schubert W. Treatment modalities for mandibular angle fractures. J Oral Maxillofac Surg. 2005 May;63(5):655-63. doi: 10.1016/j. joms.2004.02.016. PMID: 15883941.

8. Sudhakar GV, Rajasekhar G, Dhanala S, Vura N, Ramisetty S. Comparison of Management of Mandibular Angle Fractures by Three Approaches. J Maxillofac Oral Surg. 2015 Dec;14(4):979-85. doi: 10.1007/s12663-0150779-0. Epub 2015 Apr 3. PMID: 26604473; PMCID: PMC4648776.

9. Devireddy SK, Kishore Kumar RV, Gali R, Kanubaddy SR, Dasari MR, Akheel M. Transoral versus extraoral approach for mandibular angle fractures: A comparative study. Indian J Plast Surg. 2014 Sep-Dec;47(3):354-61. doi: 10.4103/0970-0358.146590. PMID: 25593420; PMCID: PMC4292112.

10. House JW, Brackmann DE. Facial nerve grading system. Otolaryngol Head Neck Surg. 1985 Apr;93(2):146-7. doi: 10.1177/019459988509300202. PMID: 3921901.

11. Ellis E 3rd. Treatment methods for fractures of the mandibular angle. Int J Oral Maxillofac Surg. 1999 Aug; 28(4):243-52. PMID: 10416889.

12. Kale TP, Baliga SD, Ahuja N, Kotrashetti SM. A comparative study between transbuccal and extra-oral approaches in treatment of mandibular fractures. J Maxillofac Oral Surg. 2010 Mar;9(1):9-12. doi: 10.1007/ s12663-010-0026-7. Epub 2010 Jun 4. PMID: 23139558; PMCID: PMC3453684.
13. Ongodia D, Li Z, Zhou H-H, Li Z-B. Comparative analysis of trends in the treatment of mandibular fractures. J Oral Maxillofac Surgery,Med,Pathol. 2014; 26:276-279.

14. Shah A, Nautiyal V, Gupta A, Ramola V. Trends of maxillofacial fractures in the Garhwal Himalayas at Government Medical College, Srinagar, Uttarakhand. Natl J Maxillofac Surg. 2016 Jan-Jun;7(1):80-85. doi: 10.4103/0975-5950.196139. PMID: 28163485; PMCID: PMC5242081.

15. Un Nisa Z, Ul Hassan Q, Hassan SG, Shams S, Khan MSU. Comparison between extraoral and intraoral surgical procedures for the treatment of mandibular angle fractures using semirigid fixation or rigid fixation. Medical Forum Monthly. 2014; 25:13-5.

16. Hsueh WD, Schechter CB, Tien Shaw I, Stupak HD. Comparison of intraoral and extraoral approaches to mandibular angle fracture repair with cost implications. Laryngoscope. 2016 Mar;126(3):591-5. doi: 10.1002/ lary.25405. Epub 2015 Jul 7. PMID: 26154627.

17. Bukhari SGA, Shahzad M, Afzal M, Ullah S, Liaqat S. Comparison between extra oral and intraoral surgical procedures for the management of mandibular angle fractures. Pak Arm Forces Med J. 2018; 68:623-26.

18. Toma VS, Mathog RH, Toma RS, Meleca RJ. Transoral versus extraoral reduction of mandible fractures: a comparison of complication rates and other factors. Otolaryngol Head Neck Surg. 2003 Feb;128(2):215-9. doi: 10.1067/mhn.2003.59. PMID: 12601317.

19. Rehman B, Iqbal A, Afsar H, Qiam ud Din, Ansari SR. Comparative analysis of extra-oral and intraoral approaches in mandibular angle fracture. JKCD. 2015; 5: 16-9. 